\title{
Change, Temporal Parts, and the Argument from Vagueness
}

\author{
Achille C. Varzi \\ Department of Philosophy, Columbia University (New York)
}

[Final version published in Dialectica 59:4 (2005), 485-498]

\begin{abstract}
The so-called "argument from vagueness", the clearest formulation of which is to be found in Ted Sider's book Four-dimensionalism, is among the most powerful and innovative arguments offered in support of the view that objects are four-dimensional perdurants. The argument is defective - I submit - and in a number of ways that is worth looking into. But each "defect", each gap in the argument, corresponds to a model of change that is independently problematic and that can hardly be built into the common-sense picture of the world. So once all the gaps of the argument are filled in, the three-dimensionalist is left with the burden of a response that cannot rely on a passive plea for common sense. The argument is not a threat to common sense as such; it is a threat to the three-dimensionalist faithfulness to common sense.
\end{abstract}

\section{Introduction}

Things change. Bananas ripen, houses deteriorate, people lose hairs and acquire new body cells. How can we say that they are the same things, if they are no longer the same? What grounds our belief that the things around us (and ourselves, too) may survive from day to day, in spite of the many changes that affect them? In this world of flux, persisting things are the only anchor we have, but the source of their persistence appears to be a genuine puzzle - a puzzle that has been with us since the Presocratics.

Today, a growing number of philosophers believe that the puzzle betrays a wrong metaphysics of persistence. Don't think of time as a special dimension along which objects continue to exist - they say. Just think of time as another dimension along which objects extend, just as they extend across the three spatial dimensions. Then the puzzle disappears. Just as an object can change in space, insofar as its spatial parts can have different properties, so it can change in time-its temporal parts can have different properties. Just as we say of a banana that it is spoiled here but not there insofar as this part of the banana is spoiled but that part isn't, we can say of the banana that it is ripe today but not yesterday insofar as today's tempo- 
ral part of the banana is ripe but yesterday's part isn't. We have got the same thing - the same banana - but its temporal parts are not quite alike, just as its spatial parts are not alike. It changes over time just as it changes over space: its change is a function of its having spatio-temporal parts that are not qualitatively identical.

This way of resetting the issue is extremely effective but also, of course, deeply revisionary. There is no question that common sense is based on a three-dimensional conception of objects - a conception according to which objects such as people and bananas, unlike events such as lives and soccer games, extend exclusively across the three spatial dimensions and persist through time by being fully present at different times - and the claim that this is a "wrong" metaphysics can hardly be taken light-heartedly. True, the scientific picture of the world seems to provide evidence in favor of the claim, at least since the formulation of special relativity theory. But for many philosophers the challenge is to make the scientific picture compatible with common sense, not to replace one for the other. So to reset the issue along the lines indicated above is to trade one problem for another: the philosophical dispute about the problem of change turns into a controversy about the metaphysical make-up of the things that change. And that is no easier question to settle.

Can it be settled? One striking feature of the recent debate on these matters is that many more arguments have been offered in favor of the revisionary, four-dimensional conception than in support of the traditional, three-dimensional view. Part of the reason is that the latter view is hardly in need of any intuitive "justification", whereas the former cries for independent evidence. The three-dimensionalist can therefore play defensively: in the absence of decisive evidence to the contrary, the traditional conception of objects as enduring continuants is all but metaphysically wrong-headed, even if it requires taking the problem of change at face value. And most threedimensionalists would insist that none of the arguments offered by their opponents is decisive enough. Still, as the arguments pile up the burden of defense gets heavier, and the three-dimensionalists can no longer satisfy themselves with the generic support that comes from the intuitive plausibility of their view. Adherence to common sense is a desideratum, not a strength.

One argument that has been put forward recently is particularly instructive in this regard, and that is what I intend to focus on in this paper. It is the so-called "argument from vagueness", the clearest formulation of which 
is to be found in Ted Sider's book Four-dimensionalism (2001). The argument is instructive because it is easy to find it defective and to rest content with such a diagnosis. However, to reject an argument as defective is to accept at least one of the countermodels that it engenders, or to reject one of the premises. For example, to reject the so-called "argument from temporary intrinsics" is to accept a model of change according to which all putative properties are in fact relations to time, or a model according to which the only properties an object has are its present properties, or a model according to which the instantiation of a property by an object is relativized to times, and so on. Three-dimensionalists may have different views regarding these models, and such differences will affect the details of their metaphysical story. But all such models are, in some important way, compatible with the common-sense picture of the world that three-dimensionalism is meant to defend. With the argument from vagueness the situation is different. The argument is defective, and in a number of ways that is worth looking into. But each "defect", I will argue, corresponds to a model of change that is independently problematic and that can hardly be built into the common-sense picture of the world. So the argument as such is not a knock-down, that is true. But once all the gaps are filled in, the challenges it raises are nonetheless serious, and one is left with the burden of a response that can no longer rely on a passive plea for common sense. The argument is not a threat to common sense as such; it is a threat to the three-dimensionalist faithfulness to common sense.

\section{The Argument from Vagueness}

Let me first review Sider's formulation. In a nutshell, the argument says that four-dimensionalism is the only plausible metaphysics of persistence unless one is willing to countenance some form of ontic indeterminacy, i.e., unless one is willing to say that there is vagueness in the world. The reason is that four-dimensionalism would afford the only way to provide the question "Under what conditions do objects come into and go out of existence?" with a plausible and definite answer. Other views - three-dimensionalism in primis - would be committed to answers that are either implausible or else irredeemably vague, and vague in a way that can only be explained in terms of ontic indeterminacy. 
Let's see how this goes in detail. Consider the following questionhenceforth the Diachronic Composition Question: Given various times and various objects existing at each, under what conditions will there be something that is composed of all and only those objects at those times? More precisely:

(Q) Given a class of time instants, $I$, and a function $f$ assigning a non-empty class of objects, $f(t)$, to each $t$ in $I$, under what conditions will there be an object, $x$, that exists exactly at the times in $I$ and that at each such time $t$ is composed exactly by the objects in $f(t)$ ?

Notice that such an object $x$, if it existed, would be a persisting object whose mereological composition at each relevant time is specified by the function $f$. So in the end the Diachronic Composition Question amounts to this: Under what conditions does $f$ identify a sequence of aggregates that add up to the career of a persisting object? In Sider's terminology: Under what conditions does $f$ have a (minimal) diachronic fusion?

Now, on the face of it, there are only three possible answers to this question:

(A1) Under no conditions at all (Nihilism)

(A2) Under certain conditions but not under others (Moderatism)

(A3) Under any conditions whatsoever (Universalism)

One might observe that, strictly speaking, nihilism is logically inconsistent unless composition is understood as strict composition, i.e., composition by proper parts. After all, every mereological atom is a fusion of itself, so when $I$ is a singleton, $\{t\}$, and $f(t)$ is itself a singleton, $\{x\}$, then there surely is an object that fits the bill, namely $x$ itself. On the other hand, with composition understood as strict composition, the nihilist answer is hardly tenable (though there are philosophers who sympathize with it-e.g. Rosen and Dorr 2002). Surely there are mereologically complex objects. My body, for example, is composed of body cells. That is, if the relevant class of times, $I$, consists of the times at which my body exists, and if the various objects existing at each such time are those body cells of mine that exist at that time, then there is something that consists of all and only those objects at those times - namely, my body. At least, this is hardly negotiable if we are interested in a comparison between three-dimensionalism and four-dimensionalism vis-à-vis common sense; a metaphysics according to which the world 
consists of time-bound mereological atoms is beyond the point. So, one way or the other, the nihilist answer is out of the game. The real alternative here is between the second answer and the third-between moderatism and universalism - and this is essentially the option between asserting some form of restricted composition versus asserting unrestricted composition. Sider argues that moderatism entails ontological vagueness - which is bad - and concludes that universalism, the only remaining option, entails four-dimensionalism.

Why should moderatism entail ontological vagueness? Intuitively, a moderate answer to the Diachronic Composition Question is certainly plausible and many philosophers have tried to specify the relevant conditions. David Wiggins (1980), for example, famously tried to come up with conditions that match up with ordinary intuitions, and many have followed in his footsteps. But - Sider argues - this is illusory. No such moderate answer can be given. That is, no moderate ontologically determinate answer can be given. Sider's reasoning here is a generalization (and correction) of a line of reasoning briefly put forward by David Lewis (1986, pp. 211ff). It comes in two parts. First, it is claimed that any restricting conditions on composition - whether synchronic or diachronic - are bound to be vague. Second, it is argued that if the conditions under which composition obtains were vague, it would be a vague matter how many objects there are, hence the vagueness in question would have to be of the ontological sort.

Concerning the first point - to the effect that any restricting conditions on composition would have to be vague - consider first synchronic composition, i.e., composition at-a-time. There is no question that we feel more at ease with certain composites than with others. We feel at ease, for instance, with the thought that there is such a thing as the fusion of all body cells that currently make up my body; but when it comes to such unlovely and gerrymandered mixtures as Lewis's (1991) trout-turkeys - those objects that consist of the front half of a trout and the back half of a turkey-we feel uncomfortable. The question is: Is there any principled way of drawing a line between those composites that we find acceptable and those that make us queasy? And Lewis's answer is in the negative: everything is queer to a degree, so any restriction on mereological composition is bound to be vague if it has to fit the intuitive desiderata. Indeed, we can easily construct a soritical series that takes us from a clear case where composition obtains (the fusion of my current body cells, the relative distance among any two of them being 
less than $n$ nanometers) to a case where composition would seem not to obtain (the fusion of my current body cells, were their relative distance increased to $n$ kilometers), and the possibility of such a construction is a sign of vagueness. With diachronic composition, says Sider, the picture ain't no better. As we have seen, there certainly is an object that at each time at which I exist consists exactly of those body cells of mine that exist at that time - namely my body. By contrast, it seems implausible to countenance objects such as Shoemaker's (1988) klables — objects that at each time in the morning consist exactly of those molecules that at that time compose a table in the kitchen and at each time in the afternoon consist exactly of the molecules that at that time compose a table in the living room. So, intuitively there are cases where composition clearly obtains (my body) and cases where composition appears not to obtain (a klable). Yet, again, there is no principled line to be drawn between the two sorts of cases. One can always come up with a series of cases any two adjacent elements of which are virtually indistinguishable, though the beginning of the series strikes us as a clear case of composition while the end makes us queasy. Whether we understand it synchronically or diachronically-the argument goes-restricted composition is prone to the sorites paradox, hence it falls prey to vagueness.

So that's the first point. Concerning the second point- to the effect that the vagueness in question would be a case of ontological vaguenessagain Sider draws on Lewis, though Sider's case is more carefully spelled out. Lewis (1986, p. 212) simply says that vagueness of composition must be ontological because the question of whether composition obtains can be stated in a portion of our language that contains no semantically vague vocabulary, but this, on the face of it, is circular. After all, the vocabulary of mereology is needed to state the question, and to assume that this vocabulary is semantically non-vague is to assume what has to be proved. Sider is more careful here: he argues instead that the question of whether composition obtains can be stated in a portion of our language that contains only logical vocabulary - quantifiers, individual variables, and identity - and no parts of the logical vocabulary are semantically vague. More precisely, Sider notes that if it ever were a vague matter whether composition obtains, then some statements of the form "There are $n$ objects" would be indeterminate in truth-value, yet their indeterminacy could not be explained away semantically because they contain no non-logical vocabulary. This means that it would be objectively indeterminate how many objects there are. And to say 
that it is objectively indeterminate how many objects there are is to say that the world is indeterminate.

Putting the pieces together, the advertised claim now follows: Any moderate answer to (Q) entails ontological vagueness. Since this sort of vagueness is unacceptable-Sider argues - there is only one option left, namely universalism. And according to Sider this is an option that only the four-dimensionalist can afford. Three-dimensionalism is of a piece with moderatism.

\section{Problems and Loose Ends}

Here, then, is the argument from vagueness in its entirety:

(1) There are three possible answers to the Diachronic Composition Question - the nihilist, moderate, and universalist answers.

(2) The nihilist answer is untenable.

(3) Any moderate answer is untenable, for:

(3.1) any such answer is bound to be vague - for

(3.1.1) it is prone to the sorites paradox, and

(3.1.2) being sorites prone is a sign of vagueness;

(3.2) the relevant vagueness is ontological-for

(3.2.1) the question of whether composition obtains can be stated in language that contains only logical vocabulary, and

(3.2.2) no parts of the logical vocabulary are semantically vague;

(3.3) ontological vagueness is untenable.

(4) Ergo, the universalist answer is the only acceptable option.

(5) This option is available to the four-dimensionalist.

(6) This option is not available to the three-dimensionalist.

(7) Ergo, four-dimensionalism affords a better metaphysics than threedimensionalism.

Why did I say that this is a defective argument? Formally it is valid, at least insofar as the two main inferences are concerned-from (1)-(3) to (4) and from (4)-(6) to (7). But it is an argument with many premises. And although Sider has done a great job to back up some of them, there is still room for several replies that are worth looking into. Let us go through them. But let us do so with an eye at the costs involved in such replies. For, eventually the question will be whether the three-dimensionalist can accept any of 
them-whether she can reject the argument and still claim faithfulness to common sense.

\subsection{The first inference}

Let us begin with the inference from (1)-(3) to (4). Premise (1) is analytic, hence uncontroversial. Premise (2) is not so straightforward, but it wears the costs of its rejection on its sleeves, at least insofar as we are interested in a comparison between three- and four-dimensionalism vis-à-vis common sense. As we have already seen, a metaphysics according to which the world consists of time-bound mereological atoms is beyond the point. So it is premise (3) that carries the burden of the inference to (4), which is why Sider himself devotes most of his efforts to a defense of this premise. We have seen that this defense takes the form of a deductive argument based on the three claims in (3.1)-(3.3). So in the end it is those claims that we have to scrutinize.

Concerning the third of these claims - to the effect that ontological vagueness is untenable-I don't have much to say. This is obviously controversial, but so be it: as with premise (2), claim (3.3) wears the costs of its rejection on its sleeves. So let us focus on the other two claims.

The obvious reply to (3.1) is that restricted diachronic composition need not be vague because, contrary to Sider's argument for (3.1.1), it need not fall pray to the sorites paradox. For one thing, one could simply insist that whether composition obtains is a brute fact (see e.g. Markosian 1998, 2004). If it is a brute fact whether composition obtains, then every numerical statement must have a definite truth-value and no soritical series could be constructed to show the vagueness of restricted composition. On the other hand, it is certainly hard to believe in brute facts on the grounds of a generic appeal to common sense. So let's put that way out aside. One could still argue as follows. What must be vague, on the face of it, is restricted composition at a time: there is no principled way of telling which mereological sums exist at a given time, for everything is queer to a degree. This means that composition-at-a-time must be fully unrestricted: given any number of objects existing at any time $t$, there is something that at $t$ is composed exactly of those objects and nothing else, whether we like it or not. But this is not to say that we are forced to accept diachronic unrestricted composition as well, i.e., the claim corresponding to the universalist answer (A3). Indeed there are 
principled ways one can rely on to support a more moderate answer corresponding to option (A2). In particular, a three-dimensionalist can rely on the criteria corresponding to the view that has come to be known as mereological essentialism (Chisholm 1973). This is the view according to which an object's parts are all essential to it. On a diachronic reading, this means that no object can gain or lose parts during its career: it is always composed of the same things. And such things can be three-dimensional: the world would consist exclusively of three-dimensional objects that are individuated by, and whose persistence conditions are given by, their three-dimensional proper parts. My body would not be such a thing, because the subatomic particles that currently compose my body are different from those that composed it yesterday. But the mereological aggregate of my current subatomic particles is such a thing. Currently it happens to be organized so as to form my body, yesterday it was somewhat more scattered, and a year ago it probably was all over the places. Yet that thing exists now as it existed yesterday and a year ago (or so we may suppose), hence at each time at which those particles exist there is something - their mereological aggregate - that exists at that time and is composed exactly of those particles, regardless of how the particles are arranged. (What we call 'my body', by contrast, is on this view a mere façon de parler, an ens successivum: different things do duty for it at different times.) So if we accept this view, then we have a perfectly clear criterion for combining unrestricted synchronic composition with restricted diachronic composition: given any number of objects existing at any given time $t$, there is something that at $t$ is composed exactly of those objects and nothing else (unrestricted synchronic composition); but given various times and various objects existing at each, a necessary and sufficient condition for there to be something that at those times is composed of those objects is that those objects be the same throughout. That is, in the precise terminology of $(\mathrm{Q})$, mereological essentialism affords the following instance of the moderate answer (A2):

(A2') Given a class of time instants, $I$, and a function $f$ assigning a non-empty class of objects, $f(t)$, to each $t$ in $I$, a necessary and sufficient condition for there to be an object that exists exactly at the times in $I$, and that at each such time $t$ is composed exactly by the objects in $f(t)$, is that $f(t)$ comprises the same objects for each $t$ in $I$.

This rules out unrestricted diachronic composition in a perfectly non-vague language. 
Now, Sider is aware of this fact, so this is not a criticism ad personam. In fact, elsewhere in his book Sider takes stock and gives his reasons for not liking mereological essentialism precisely because "it is a three-dimensional view that is untouched by my [Sider's] argument from vagueness for fourdimensionalism" (p. 180). But never mind that. Whatever reasons a fourdimensionalist such as Sider may have to reject mereological essentialism, what matters here is that a mereological essentialist can reject (3.1) and block the argument at this juncture - and mereological essentialism embodies a three-dimensionalist metaphysics. On the other hand, it is certainly appropriate to ask whether this is the sort of metaphysics that a three-dimensionalist is allowed to offer vis-à-vis the common-sense picture of the world that she aims to defend. And here the answer strikes me as obviously negative. As many philosophers have argued, the thought that ordinary objects such as my body or Theseus's ship are just entia successiva is certainly at odds with common sense. One is free to go along with it if one deems the other options unacceptable. But the grounds for such choice must be put on the table: faithfulness to common sense is of no help in this context, just as it is of no help if one decides to reject (3.1) by appealing to brute facts.

This leaves us with (3.2), namely with the claim that the vagueness of restricted diachronic composition must be of the ontological sort. As we have seen, Sider's point is that vague composition implies the vagueness of our logical vocabulary, as per (3.2.1). But why should the latter be a sign of ontological vagueness? Here there is room for two sorts of misgiving. On the one hand, one could reject claim (3.2.2), to the effect that no parts of the logical vocabulary are semantically vague; on the other, one could accept that claim and yet deny that the alternative here is between semantic and ontological vagueness.

Let us begin from the second option. Consider a statement of the form "There are $n$ objects" and suppose that this statement lacks a definite truthvalue - it is neither determinately true nor determinately false. Even assuming the semantic determinacy of the vocabulary, one need not attribute the relevant indeterminacy to the way the world is (or isn't). One could cash out the relevant indeterminacy in epistemic terms. That is, one could say that it is epistemically indeterminate how many objects there are: given various times and various objects existing at each, either there is something that is composed of all and only those objects at those times, or there isn't-we just cannot find out. I am not thinking here of an epistemicist account. In his 
book, Sider considers that possibility and rightly points out that the epistemicist account of vagueness does not sit well with the case at issue. As Roy Sorensen (1988), Tim Williamson (1994), and other defenders have stressed, the sharp cut-off points postulated by the account are not meant to correspond to unexpected joints in reality. Rather, they would represent unanticipated powers of language users to draw what appear to be metaphysically arbitrary lines. Somehow, something about our meaning-fixing behavior determines a sharp cut-off point where there seem to none- a cut-off that we are not in a position to identify. Whether this is a plausible account is irrelevant here. What is relevant is that if it works, it only works for the indeterminacy of the non-logical vocabulary - the indeterminacy of predicates such as 'bald' and of names such as 'Everest'. When it comes to the logical vocabulary, it is no longer a matter of use-meaning bridge laws. The existence of a sharp cut-off would have to call for a metaphysical explanation, and that goes beyond the scope of the epistemicist account. So, what I have in mind when I speak of an epistemic account of the indeterminacy of a numerical statement such as "There are $n$ objects", which consists exclusively of logical vocabulary, is something else. It is genuine metaphysical ignorance. It feels metaphysically arbitrary to draw the line somewhere and there is no way to settle the matter, so it is impossible to attach a definite truth-value to our numerical statement. Yet we should not blame the world for such ignorance: the world is determinate; it's our metaphysical dexterity that is limited. (I think this is close to the view held by Hudson 2000.)

This way of rejecting the inference to (3.2), and therefore to (3), strikes me as intelligible. But it does come at a price, too-namely, it embodies a non-realist conception of truth that is by itself highly controversial. Indeed, in order for the reply to go through, we need to accept a conception of truth as superassertibility, or something along those lines. For only such a conception would allow us to say that the impossibility to attach a truthvalue to a statement entails that the statement itself lacks a truth-value. On a realist conception, ignorance has nothing to do with truth and falsity. If there is a determinate number of objects, then on a realist conception of truth every numerical statement has a definite truth-value, whether or not we are capable of computing it - and this takes us back to the "brute facts" way out mentioned above.

Consider now the other option. As I mentioned, this consists in rejecting (3.2) by explicitly rejecting (3.2.2), namely the claim that no parts of 
the logical vocabulary are semantically vague. Sider argues that this would be wrong-headed because it would amount to the claim that the logical operators, among which the quantifiers, admit of multiple precisifications, when in fact they do not. Sider's reasons for this claim, however, are formally questionable. Let's focus on the quantifiers, which is really what matters in the present context. What is wrong with the thought that they admit of multiple precisifications? Suppose there were two precisifications of the existential quantifier, $\mathrm{P} 1$ and $\mathrm{P} 2$, which differ in their range. Then there would have to be something, $x$, that is in the range of one, say $\mathrm{P} 1$, but not in the range of the other, P2. Sider claims that this is impossible for the following reason: since P2 lacks $x$ in its range, it fails to be an acceptable precisification of the unrestricted universal quantifier. "It quite clearly is a restricted quantifier since there is something - $x$ - that fails to be in its extension" [p. 129]. This is incorrect, and for a reason that Sider himself considers and yet rejects. The reason is that the phrase 'there is' - as it occurs in the above quotation-is ambiguous. Why should we take it to range over a domain that includes $x$ ? To do so would be to identify this phrase with the existential quantifier as precisified by P1. But why so? On pain of circularity, there is no "Archimedean point"- as Sider himself puts it-from which to claim that P2 is a restricted quantifier. There is genuine competition between P1 and P2, and this competition affects the meaning to be attached to the phrase 'there is'. Sider says that it is hard to understand what these precisifications are supposed to be, but on the face of it there is a perfectly clear semantic model on the table: broadly understood, supervaluationism is in no way committed to the thought that the domain of quantification must be fixed as we go from one precisification to another (see Skyrms 1968.)

I think this line of objection should not be underestimated. Once again, however, the question that concerns us here is not merely the existence of a weak spot in the vagueness argument. The question is whether this spot represents a weakness in the argument that a three-dimensionalist can delve into and build on. For what is the price of this move? On the one hand, to say that the quantifiers are vague is to say that there is vagueness in the ontologically significant portion of our language, and to many philosophers this is just a different way of saying that there is ontic vagueness. On the other hand, one could cash out the relevant vagueness in terms of competing conceptual schemes. Different precisifications would correspond to different schemes - different models of the world. But unless one takes this to mean 
that all quantification is restricted insofar as each conceptual scheme is partial - a claim that would leave no room for the sort of quantification that deserves a place in the logical vocabulary - the consequence of this view would be a radical form of relativism according to which what there is depends on what we think and how we talk. That is not an option that can be ruled out $a$ priori-many philosophers sympathize with this sort of relativism (with various qualifications, as in Sosa 1999 or Hirsch 2002). But it certainly is not an option that can be justified by mere appeal to common sense.

\subsection{The second inference}

So much for the first half of the argument. Let us now move to the second, namely the inference from (4)-(6) to (7). Assuming universalism is indeed the only acceptable answer to the Diachronic Composition Question, what justifies the twofold claim that this answer is available to the four-dimensionalist (premise (5)) but not to the three-dimensionalist (premise (6))?

That the four-dimensionalist has this option is, I think, uncontroversial. Since Quine (1950), four-dimensionalism has often been formulated precisely as a view to the effect that any filled region of space-time is the total career of some object, indeed that any filled region of space-time is an object. So premise (5) is all right. But what about (6)? What prevents a threedimensionalist from holding a parallel view? Note that Sider is very careful in phrasing $(\mathrm{Q})$ in such a way as not to beg the question. The universalist answer (A3) does not say that, given various times and various objects existing at each, there is sure to exist a diachronic mereological fusion of all those objects. It says that, given various times and various objects existing at each, there is sure to be something that exists at each of those times and that at each such time is composed of the corresponding objects. In other words, the universalist answer corresponding to (A3) is just a temporalized version of a principle of plenitude concerning the existence of mereological fusions. But as Kathrin Koslicki (2003) and Jonathan Lowe (2005) have pointed out, there is no obvious reason why such a temporalized plenitude principle cannot be accepted (on a suitable understanding of 'temporalized') by a mereologically promiscuous three-dimensionalist as well. Judith Thomson (1983), for instance, is such a philosopher.

On my reckoning, this is actually the strongest reply one could put forward against the argument from vagueness as a whole. For even granting 
the inference from (1)-(3) to (4) - on which most of the debate has focused - it simply is not clear why one should take the case for universalism to be a case for four-dimensionalism. Surprisingly, Sider says nothing explicit about this in his book. And in his 2003 reply to Koslicki, he only offers a parenthetic remark: by implying the existence of arbitrary minimal diachronic fusions, universalism implies the existence of instantaneous objects (among other things) - entities which three-dimensionalists like Thomson reject. This may well be true in respect of Thomson. But I don't see why a promiscuous three-dimensionalist should in principle be opposed to countenancing instantaneous objects as a special case. Even if we stress the importance of common sense, as we have been doing so far, I don't see any reason to think that such momentary entities should be deemed repulsive. Some things last long, some last for a short time. Some might just last the span of an instant. Sider adds: "I suspect that most [three-dimensionalists] would react thus: "If I needed to accept that (ubiquitous instantaneous objects), I might as well concede any remaining differences and accept four-dimensionalism"" (2003, p. 136). I see the point, but mostly as a rhetorical move. For all that has been said, the three-dimensionalist may not concede the remaining differences and block the argument right at this juncture.

Still, suppose a three-dimensionalist is willing to go this far. Suppose she is willing to accept the promiscuous ontology required by her adherence to (A3), with instantaneous objects along with all sorts of gerrymandered persisting beasts. The outcome, I claim, would be a metaphysics that is committed either to contingent identity or else to massive co-location-and these are views that cry for justification. For consider what happens. Let $t_{1}$ and $t_{2}$ be two distinct times, and consider two different assignments, $f$ and $f^{\prime}$, such that $f\left(t_{1}\right)=f^{\prime}\left(t_{1}\right)$ and $f\left(t_{2}\right) \neq f^{\prime}\left(t_{2}\right)$. For instance, $f\left(t_{1}\right)$ and $f^{\prime}\left(t_{1}\right)$ could be the class of those body cells of mine that exist at a given time, $t_{1}$, whereas $f\left(t_{2}\right)$ and $f^{\prime}\left(t_{2}\right)$ could comprise those cells that compose my body and Ted Sider's body, respectively, a moment later, at $t_{2}$. Intuitively, this is a scenario in which a certain entity, $a$, changes from $t_{1}$ to $t_{2}$ by replacing my earlier body cells with my later body cells, and a certain entity, $b$, changes from $t_{1}$ to $t_{2}$ by replacing my earlier body cells with Sider's later body cells. What are we to say of such entities, $a$ and $b$ ? For the four-dimensionalist, the answer is straightforward. We have two persisting entities, two distinct fourdimensional worms that have different temporal parts and occupy different regions of space-time, though at $t_{1}$ they occupy the same region of space and 
have the same spatial parts. There is nothing special about this, just as there is nothing special about the fact that the left-branching portion of a Y-shaped object, for example, partially overlaps (at the bottom) its right-branching portion: two things with a common part. The three-dimensionalist cannot put it this way, though, because she rejects temporal parts and, with them, the analogy between space and time that makes the four-dimensionalist account so straightforward. For a three-dimensionalist, $a$ and $b$ are entities that exist in their entirety at $t_{1}$ and continue to exist in their entirety at $t_{2}$. So how are they related?

There are two options. The first is to say that $a$ and $b$ are identical at $t_{1}$ (for they are composed exactly of the same parts and occupy exactly the same region of space) but distinct at $t_{2}$ (they are composed of different parts and occupy different regions of space). This is to accept contingent identity, or rather "occasional identity" (Gallois 1998), a view that can hardly be attributed to common sense. Alternatively, the three-dimensionalist can say that $a$ and $b$ are distinct not only at $t_{2}$, but also at $t_{1}$. That is, at $t_{1}$ they are distinct though fully coincident, both mereologically and spatially. This does away with contingent identity, but it means that the mereologically promiscuous three-dimensionalist is forced to give up an important principle that is often taken to be part and parcel of common sense - the Lockean principle of identity in terms of spatial location. In fact, this is just the beginning. For of course, not only would we have two things, $a$ and $b$, perfectly coinciding at $t_{1}$. On this view, there would be lots of things perfectly coinciding at $t_{1}$ : one for each possible assignment $f_{k}$ such that $f_{k}\left(t_{1}\right)=f\left(t_{1}\right)$. And on any reasonable count, there are uncountably many such assignments. To put it vividly suppose $t_{1}$ is the present instant. As you are looking at these body cells of mine, you would in fact be looking at uncountably many things - things that are indiscernible and yet would differ from one another by virtue of differences that will manifest themselves at a later time. They would differ because different are the things they will turn into (whereas on the fourdimensional account you would of course be looking at just one thing, exactly as you would be looking at one thing were you looking at the bottom portion of a Y-shaped object). Even more vividly consider the career of my body from time $t_{1}$ to time $t_{2}$. During this interval, my body undergoes mereological change, so let $f\left(t_{i}\right)$ be the class of body cells constituting my body at $t_{i}$, for each $t_{i}$ between $t_{1}$ and $t_{2}$ included. Surely the thought that $f$ has a diachronic fusion, call it $x$, is germane to the common-sense picture of change. 
But in addition to $x$, universalism implies the existence of a continuum of entities whose career is progressively shorter than that of $x$, up to the instantaneous entity that exists only at $t_{1}$. All of these entities are fully present at each time at which they exist and yet distinct. And since they all exist at $t_{1}$, at $t_{1}$ we must acknowledge the existence of continuously many, perfectly colocated things. Consistent as it might be, this picture of change certainly strikes me as one that a three-dimensionalist can hardly accept. At least, it strikes me as a picture that cries for a metaphysical justification that common sense cannot deliver.

\section{Conclusion}

Let us wind things up. Here is the list of all the options we have considered to block the "vagueness argument":

(a) Reject premise (2) and accept nihilism.

(b) Reject premise (3) by rejecting (3.1); this amounts to accepting either brute facts (contrary to (3.1.1)) or else some radical view such as mereological essentialism (also contrary to (3.1.1)).

(c) Reject premise (3) by rejecting (3.2); this amounts to accepting either a non-realist account of truth (if (3.2.2) is accepted) or a radical form of relativism (if (3.2.2) is rejected).

(d) Reject premise (3) by rejecting (3.3); this amounts to accepting plain ontological vagueness.

(e) Reject premise (6) and accept either contingent identity or massive colocation.

On my reckoning, these are the only options. They are not few, and for this reason the argument is not a straight knock-down in favor of fourdimensionalism. But neither are these options of a piece with common sense. On the contrary, some of them are quite at odds with the pre-analytic picture of the world that three-dimensionalism is meant to reflect. If this is right, then it seems to me that the defensive strategy of the threedimensionalist has better improve. Adherence to common sense is a desideratum. But something has to give, and it is by no means obvious that the price to be paid by a three-dimensionalist will be inferior to the revisionary move so kindly advocated by four-dimensionalists.

\footnotetext{
* Many thanks to Fabrice Correia and Philipp Keller for their comments on earlier versions.
} 


\section{References}

Chisholm, R. M. (1973), 'Parts as Essential to Their Wholes', Review of Metaphysics 26: 581-603.

Gallois, A. (1998), Occasions of Identity: The Metaphysics of Persistence, Change, and Sameness, Oxford: Clarendon Press.

Hirsch, E. (2002), 'Quantifier Variance and Realism', Philosophical Issues 12: $51-73$

Hudson, H. (2000), 'Universalism, Four-Dimensionalism, and Vagueness', Philosophy and Phenomenological Research 60: 547-560.

Koslicki, K. (2003), 'The Crooked Path From Vagueness to Four-Dimensionalism', Philosophical Studies 114: 107-134.

Lewis, D. K. (1986), On the Plurality of Worlds, New York: Blackwell.

Lewis, D. K. (1991), Parts of Classes, New York: Blackwell.

Lowe, E. J. (2005), 'Vagueness and Endurance', Analysis 65: 104-112.

Markosian, N. (1998), 'Brutal Composition', Philosophical Studies 92: 211249 .

Markosian, N. (2004), 'Two Arguments from Sider's Four-Dimensionalism', Philosophy and Phenomenological Research 68: 665-673.

Quine, W. V. O. (1950), 'Identity, Ostension, and Hypostasis', Journal of Philosophy 47: 621-633.

Rosen, G., and Dorr, C. (2002), 'Composition as a Fiction', in R. Gale (ed.), The Blackwell Guide to Metaphysics, Oxford: Blackwell, pp. 151-174.

Shoemaker, S. (1988), 'On What There Are', Philosophical Topics 16: 201223.

Sider, T. (2001), Four-Dimensionalism: An Ontology of Persistence and Time, Oxford: Clarendon.

Sider, T. (2003), 'Against Vague Existence', Philosophical Studies 114: 135146.

Skyrms, B. (1968), 'Supervaluations: Identity, Existence, and Individual Concepts', The Journal of Philosophy 69: 477-482.

Sorensen, R. A. (1988), Blindspots, Oxford: Clarendon Press.

Sosa, E. (1999), 'Existential Relativity', Midwest Studies in Philosophy 23: 132-143.

Thomson, J. J. (1983), 'Parthood and Identity Across Time', Journal of Philosophy 80: 201-220.

Wiggins, D. (1980), Sameness and Substance, Oxford: Blackwell.

Williamson, T. (1994), Vagueness, London: Routledge. 